\title{
NR32: REVISÃO À PROTEÇÃO EM LABORATÓRIOS DE INSTITUIÇÕES DE ENSINO SUPERIOR
}

\section{Bruno Veloso Fracasso}

brunofracasso@hotmail.com Universidade Federal de Ciências da Saúde de Porto Alegre UFCSPA, Porto Alegre, Rio Grande do Sul, Brasil

Cláudia de Souza Libânio claudiası@ufcspa.edu.br Universidade Federal de Ciências da Saúde de Porto Alegre UFCSPA, Porto Alegre, Rio Grande do Sul, Brasil

Fernando Gonçalves Amaral amaral@producao.ufrgs.br Universidade Federal do Rio Grande do Sul - UFRGS, Porto Alegre, Rio Grande do Sul, Brasil

\section{RESUMO}

Destaques: $\mathrm{O}$ trabalhador da área da saúde está em uma das categorias profissionais que mais apresentam riscos de sofrer acidentes laborais ou de contraírem patologias em função da sua atividade profissional. Estes têm na Norma Regulamentadora 32 as diretrizes que propõe condições de trabalho seguras e que devem ser seguidas em todo país.

Objetivo: compreender o cenário atual dos laboratórios de saúde de instituições de ensino superior referente à saúde e segurança no trabalho (SST).

Desenho/Metodologia/Abordagem: para o atingimento do objetivo, optou-se por estruturar uma pesquisa do tipo revisão de literatura narrativa.

Resultados: foram encontrados 12 artigos que estavam relacionados com o tema da pesquisa, entretanto nenhum deles tratando do uso da norma em laboratórios de instituições de ensino superior, especificamente.

Limitações da investigação: Não foi localizado nenhuma pesquisa ao qual o objeto de estudo fosse a NR32 em laboratórios de saúde de instituições de ensino superior, logo, os achados do presente artigo devem ser compreendidos e transferidos para a realidade encontrada por professores, técnicos e alunos.

Implicações práticas: através dos achados será possível o desenvolvimento de cultura de saúde e segurança ocupacional, desde o momento da formação de futuros profissionais. Originalidade/valor: a ausência de material científico que explore o tema saúde e segurança no trabalho em laboratórios de instituições de ensino mostra o quão relevante e valioso tal tema apresenta.

Palavras-chaves: Norma Regulamentadora 32; saúde e segurança do trabalho; laboratórios. 


\section{INTRODUÇÃO}

Quando se fala em saúde dos trabalhadores da área da saúde, tem-se em mente que os profissionais dessa área não possuem problemas relevantes na sua rotina laboral, uma vez que se encontram inseridos em ambientes que cuidam da saúde e até trabalham com a promoção da saúde de forma geral (ABEn, 2006). No entanto, diversas categorias de profissionais da saúde têm apresentado doenças ocupacionais e se envolvido em acidentes de trabalho. São vários os fatores que levam esses profissionais ao acometimento de doenças laborais. Entre esses fatores colaboradores para o adoecimento dos trabalhadores da saúde estão a jornada extensa de trabalho a que muitos se submetem, sobretudo quando se trata da assistência ininterrupta ao enfermo. Outro fator que justifica os casos de problemas de saúde pode estar relacionado com o fato desses profissionais estarem sempre em contato físico direto com os pacientes (ABEn,2006).

É importante destacar que o espaço hospitalar é classificado pela legislação brasileira como um ambiente que faz parte do setor terciário, sendo reconhecido como ambiente com grau três de risco. Essa classificação é feita em razão das atividades que ali são desenvolvidas serem consideradas como insalubres (Marziale; Carvalho, 1998). Por essa razão, o Ministério do Trabalho e Emprego (MTE), com a criação NR32, estabelece os protocolos e medidas que estão a favor da segurança e proteção dos profissionais da saúde, incluindo também os trabalhadores da área de ensino e pesquisa.

Na norma regulamentadora são explicitados os principais riscos a que o trabalhador fica exposto. Acácio et al. (2013) trazem que para efeito desta NR, consideram-se riscos ambientais os agentes físicos, químicos e biológicos existentes nos ambientes de trabalho que, em função de sua natureza, concentração ou intensidade e tempo de exposição, são capazes de causar danos à saúde do trabalhador.

Na NR32 são destacados os riscos que comumente são associados ao adoecimento dos trabalhadores, como os agentes de risco químico, risco biológico, risco ergonômico e os agentes de risco físico, onde são destacadas as radiações ionizantes. A norma prevê que todos os trabalhadores devem ter assegurada capacitação quanto aos riscos expostos antes do início das atividades e de forma continuada sempre que ocorra uma mudança das condições de exposição ao agente (Brasil, 2005).

Dessa forma, este artigo analisará pesquisas científicas sob a perspectiva da NR32, buscando compreender o cenário atual dos laboratórios de saúde de instituições de ensino superior referente à saúde e segurança no traba-
Iho (SST). Para tanto, será utilizada a revisão bibliográfica narrativa, através de literatura científica dos últimos 10 anos.

\section{METODOLOGIA}

O enfoque do presente trabalho concentra-se na análise e avaliação dos artigos científicos que abordam a Norma Regulamentadora 32. Essa análise terá como foco a percepção que os autores dos artigos apresentam sobre a NR32, ressaltando os objetivos e resultados alcançados com as pesquisas.

Para tanto, busca-se a utilização de fontes bibliográficas que discutem o tema, haja vista que a análise das obras de autores diversos pode contribuir para a variedade de ideias acerca do tema. Corroborando com essa afirmativa, Marconi e Lakatos (2003) afirmam que a pesquisa bibliográfica implica em um processo teórico que tem por objetivo levantar informações sobre determinada temática.

A abordagem utilizada para o estudo teve como foco a pesquisa qualitativa por meio de uma revisão bibliográfica narrativa. Chizzotti (2003) afirma que a abordagem qualitativa concentra sua análise nos dados e informações que tem algum significado para os indivíduos. Neste tipo de pesquisa busca-se compreender o sentido das ações, compreendendo as interações entre essas ações e o contexto em que se manifestam.

A pesquisa baseou-se nos pressupostos da pesquisa bibliográfica, sendo entendida como o apanhado teórico acerca do tema, constituído no fornecimento de dados atuais e relevantes para o trabalho. Nesta pesquisa foram consultados artigos científicos em saúde e segurança do trabalho em instituições de saúde, que contribuem, sobremaneira, para a temática da pesquisa. Para fins de pesquisa, seguiu-se o item 32.1.2 da NR32 onde traz que:

Para fins de aplicação desta NR entende-se por serviços de saúde qualquer edificação destinada à prestação de assistência à saúde da população, e todas as ações de promoção, recuperação, assistência, pesquisa e ensino em saúde em qualquer nível de complexidade (Brasil, 2005).

Para a pesquisa em questão foram consultados artigos científicos de algumas bases de dados como Scientific Eletronic Library Online (SciELO), além de dados e informações provenientes da busca na Biblioteca Virtual em saúde (BVS) e Google Acadêmico com o uso do descritor "norma regulamentadora 32". 


\section{RESULTADOS E DISCUSSÃO}

A NR32 tem como foco a normatização, regulamentação dos acidentes e casos de adoecimento que surgem nos espaços de trabalho dos trabalhadores da saúde, levando em conta, principalmente, os riscos a que esses profissionais estão sujeitos no seu ambiente de trabalho. Silva (2018) esclarece que:

com o advento da Portaria n. 485 (DOU 16.11.05) que introduziu a Norma Regulamentadora 32 (NR32), institui-se que seu objetivo e finalidade é estabelecer as diretrizes básicas para implementação de medidas de proteção à saúde dos trabalhadores dos serviços de saúde, bem como daqueles que exercem atividades de promoção e assistência à saúde em geral.

De forma geral, a NR32 trabalha com a eliminação ou controle dos riscos que estão presentes nos locais que oferecem serviços de saúde. A norma especifica que o controle desses riscos deve ser solidário, sendo divididas as responsabilidades entre empregado e empregador. Isso ressalta a importância de que os envolvidos na oferta dos serviços de saúde devem se atentar para as medidas de segurança a serem seguidas no ambiente (Brasil, 2005)

Em um documento da Anvisa (2003) fica explícita a definição de risco, quando o documento afirma que risco é a "probabilidade de possíveis danos dentro de um período de tempo ou número de ciclos operacionais".

No ambiente de trabalho dos profissionais da saúde é possível encontrar diversas situações que trazem riscos para a saúde desses trabalhadores. Comumente, observa-se que os riscos ocorrem em função da falta ou não utilização dos EPIs, uso de vestimentas e calçados inadequados, porte de adereços que podem facilitar o fluxo de contaminação, além do acondicionamento incorreto de seringas e objetos cortantes que facilitam a contaminação de micro-organismos pelo espaço hospitalar (Robazzi; Marziale, 2004. Pensando na diminuição desses riscos, a legislação trabalhista conta com a NR32 que oferece subsídios para intervir nas situações de riscos dentro do ambiente hospitalar, oferecendo também os protocolos e medidas que auxiliam na prevenção dos riscos ocupacionais para os trabalhadores da saúde.

A NR32 é considerada como a norma que regulamenta e norteia todo o desenvolvimento das atividades laborais dos trabalhadores da saúde. Ela é responsável também por explanar sobre os riscos a que esses profissionais estão suscetíveis, esclarecendo de forma específica sobre os procedimentos e protocolos que devem ser seguidos, para que se consiga a redução dos riscos inerentes a profissões dessa área (Brasil, 2005).

Para que ocorra de fato a diminuição dos riscos nas atividades de trabalho em ambientes de saúde, a NR32 prevê que seja utilizado um protocolo de atividades que tem como base legal a NR32 (Brasil, 2005).

A análise realizada nos artigos científicos possibilitou que encontrássemos 12 artigos que estavam relacionados com o tema da pesquisa, entretanto nenhum deles tratando do uso da norma em laboratórios, especificamente. $\mathrm{Na}$ tentativa de mostrar os resultados que foram obtidos, optou-se por apresentar os artigos analisados através do Quadro 01, que apresenta alguns dados sobre os títulos dos artigos, autoria(s), ano em que foram publicados, nome dos periódicos, além de especificar o objetivo do estudo e a abordagem apresentada em cada uma das pesquisas.

Os artigos utilizados para a revisão de literatura foram escolhidos obedecendo a critérios, tais como proximidade com o assunto proposto e periódico em que foi publicado e ano de publicação, sendo respeitado o limite máximo de 10 anos de publicação. A escolha de determinados artigos respondeu ao objetivo proposto pela pesquisa, uma vez que representava de forma explícita a importância da NR32 nos ambientes laborais dos profissionais da saúde.

Em alguns desses artigos foi possível perceber uma ênfase maior em relação aos riscos à saúde quando não ocorria a utilização da NR32 dentro dos espaços de saúde, implicando no desenvolvimento não seguro das atividades de trabalho. No entanto, percebeu-se também que alguns artigos tinham como objeto a discussão sobre a necessidade de implantação da NR32, no sentido de apresentar o impacto dos protocolos de prevenção e diminuição de riscos para os profissionais da saúde. Oliveira et al. (2015) afirmam que "desenvolver ações de educação em saúde, discutindo biossegurança e precaução padrão é uma das primeiras ações necessárias para a prevenção e controle dos acidentes, além da adoção de medidas preconizadas na NR32".

Marziale et al. (2012) ao buscarem identificar os acidentes de trabalho com exposição à material biológico ocorridos em um hospital universitário, evidenciaram que o percentual de acidentes de trabalho reduziu ao longo do período, no qual diversas exigências da norma foram adotadas. Entretanto, Silva et al. (2015) salientam que é necessário compreender que tanto as diretrizes quanto o compromisso não são unilaterais, mas sim que ocorrem entre as instituições de saúde e os profissionais. É necessário que o movimento envolva aspectos relacionados a oferta de uma estrutura adequada, materiais disponíveis, 
Quadro 1. Lista de autores, ano de publicação e objetivo das pesquisas.

\begin{tabular}{|c|c|c|c|}
\hline Título & Autores & Ano & Objetivo \\
\hline $\begin{array}{l}\text { Diagnóstico da implantação e implementação } \\
\text { da norma regulamentadora } 32 \text { nos estabeleci- } \\
\text { mentos de saúde: um estudo de caso em um } \\
\text { hospital público de Joinville, SC - Brasil }\end{array}$ & $\begin{array}{l}\text { Clock D, } \\
\text { Batiz EC }\end{array}$ & 2017 & $\begin{array}{l}\text { Diagnosticar a implantação e implementação da NR32 nos } \\
\text { estabelecimentos de saúde. }\end{array}$ \\
\hline $\begin{array}{l}\text { Conhecimentos e condutas de biossegurança } \\
\text { entre docentes de enfermagem }\end{array}$ & $\begin{array}{l}\text { Morais } \\
\text { RLGL et } \\
\text { al. }\end{array}$ & 2017 & $\begin{array}{l}\text { Descrever o conhecimento dos docentes enfermeiros de um } \\
\text { curso de enfermagem sobre Norma Regulamentadora } 32 \text { e as } \\
\text { condutas pós-exposição a materiais biológicos e identificar a } \\
\text { situação vacinal destes docentes. }\end{array}$ \\
\hline $\begin{array}{l}\text { Norma regulamentadora } 32 \text { no Brasil: revisão } \\
\text { integrativa de literatura }\end{array}$ & $\begin{array}{l}\text { Santos } \\
\text { Junior } \\
\text { AG et al. }\end{array}$ & 2015 & $\begin{array}{l}\text { Conhecer as principais publicações na literatura sobre a nor- } \\
\text { ma regulamentadora } 32 .\end{array}$ \\
\hline $\begin{array}{l}\text { Relato de experiência do treinamento em } \\
\text { serviço } \\
\text { Sobre a norma regulamentadora - NR32 }\end{array}$ & $\begin{array}{l}\text { Silva VG } \\
\text { et al. }\end{array}$ & 2015 & $\begin{array}{c}\text { Relatar a experiências de } 03 \text { (três) docentes de enfermagem } \\
\text { na divulgação da Norma Regulamentadora número 32, em } \\
\text { parceria com a Educação Continuada de um Hospital Munici- } \\
\text { pal do município do Rio de Janeiro. }\end{array}$ \\
\hline $\begin{array}{l}\text { Acidentes com perfurocortante entre trabalha- } \\
\text { dores de saúde }\end{array}$ & $\begin{array}{l}\text { Oliveira } \\
\text { JS et al. }\end{array}$ & 2015 & $\begin{array}{l}\text { Identificar a produção científica sobre acidentes com perfuro- } \\
\text { cortante relacionados aos trabalhadores de saúde. }\end{array}$ \\
\hline $\begin{array}{l}\text { Modelo e-learning de capacitação na Norma } \\
\text { Regulamentadora no } 32 \text { (NR32) }\end{array}$ & $\begin{array}{l}\text { Pusti- } \\
\text { glione M } \\
\text { et al. }\end{array}$ & 2015 & Propor um modelo de capacitação. \\
\hline $\begin{array}{c}\text { Consequências da exposição ocupacional a } \\
\text { material biológico entre trabalhadores de um } \\
\text { hospital universitário }\end{array}$ & $\begin{array}{l}\text { Marziale } \\
\text { MHP et } \\
\text { al. }\end{array}$ & 2014 & $\begin{array}{c}\text { Teve como objetivo analisar a ocorrência, as características } \\
\text { e consequências do acidente de trabalho com exposição a } \\
\text { material biológico para trabalhadores e instituição. }\end{array}$ \\
\hline $\begin{array}{l}\text { Aplicabilidade da Norma Regulamentadora } \\
32 \text { (NR32) e implicações para o enfermeiro do } \\
\text { trabalho }\end{array}$ & $\begin{array}{l}\text { Acácio } \\
\text { AJ et al. }\end{array}$ & 2013 & $\begin{array}{l}\text { Analisar os fatores que interferem na aplicabilidade da NR32 } \\
\text { por parte do enfermeiro do trabalho. }\end{array}$ \\
\hline $\begin{array}{c}\text { Saúde do trabalhador e riscos de resíduos no } \\
\text { ambiente hospitalar segundo a Norma Regula- } \\
\text { mentadora } 32(10)\end{array}$ & $\begin{array}{l}\text { Cremer } \\
\text { E et al. }\end{array}$ & 2013 & $\begin{array}{l}\text { Objetivou o levantamento dos fatores de risco ocupacionais a } \\
\text { que estão expostos os trabalhadores do ambiente hospitalar, } \\
\text { realizado em um hospital filantrópico do interior do Paraná. }\end{array}$ \\
\hline $\begin{array}{l}\text { Implantação da Norma Regulamentadora } 32 \text { e } \\
\text { o controle dos acidentes de trabalho }\end{array}$ & $\begin{array}{l}\text { Marziale } \\
\text { MHP et } \\
\text { al. }\end{array}$ & 2012 & $\begin{array}{l}\text { Identificar os acidentes de trabalho com exposição à material } \\
\text { biológico ocorridos em um hospital universitário. }\end{array}$ \\
\hline $\begin{array}{l}\text { Avaliação da implantação da Norma Regula- } \\
\text { mentadora } 32 \text { em um hospital universitário }\end{array}$ & $\begin{array}{l}\text { Santos } \\
\text { MR et al. }\end{array}$ & 2012 & $\begin{array}{l}\text { Teve como objetivo verificar a implantação da Norma Regula- } \\
\text { mentadora } 32 \text { em um hospital do Norte do Paraná. }\end{array}$ \\
\hline $\begin{array}{l}\text { Educação continuada e a Norma Regulamenta- } \\
\text { dora 32: utopia ou realidade na Enfermagem? }\end{array}$ & $\begin{array}{l}\text { Cunha } \\
\text { AC, } \\
\text { Mauro } \\
\text { MYC }\end{array}$ & 2010 & $\begin{array}{l}\text { Descrever o treinamento oferecido pelo Programa de Educa- } \\
\text { ção Continuada na instituição, segundo os trabalhadores de } \\
\text { enfermagem, e analisar como este treinamento pode influen- } \\
\text { ciar na implantação da NR32 no hospital. }\end{array}$ \\
\hline
\end{tabular}

condições dignas de trabalho, da mesma forma que é necessária uma atuação dos trabalhadores com responsabilidade e respeito às diretrizes propostas. Oliveira et al. (2015) ponderam que os acidentes com perfurocortante geram impactos negativos tanto para os trabalhadores quanto para as empresas, uma vez que tais episódios podem gerar afastamento destes, além do abalo emocional e psíquico que o tratamento pós-acidente pode causar.

O trabalho Cremer et al. (2013) objetivou realizar o levantamento dos fatores de risco ocupacionais a que estão expostos os trabalhadores do ambiente hospitalar e constatou que diversas unidades apresentaram riscos de resíduos, permitindo recomendar propostas de intervenções nos serviços de saúde para minimização dos riscos existentes. Marziale et al. (2014) tiveram como objetivo analisar a ocorrência, as características, consequências do acidente de trabalho com exposição a material biológico para trabalhadores e instituição. Demonstraram que a maioria dos trabalhadores acidentados eram mulheres $(94,6 \%)$, sendo auxiliares de enfermagem $(67,8 \%)$ e que grandes partes dos acidentes ocorreram na realização de punção venosa e administração de medicamentos com a manipulação de agulhas sem dispositivo de proteção. 
O mesmo estudo ainda aponta que as consequências de um acidente de trabalho com material biológico geram consequências às instituições também, uma vez que estas sofrerão com absenteísmo, necessidade de reorganização do trabalho e os prejuízos financeiros.

Acácio et al. (2013) ao analisarem os fatores que interferem na aplicabilidade da NR32, concluem que o treinamento e formação específica na NR32 são quesitos estratégicos de implantação de medidas de proteção à saúde do trabalhador. Pustiglione et al. (2015), atentos à NR32, nos que tange os aspectos de treinamento e oferta de informações sobre os agentes de risco, propuseram um modelo de capacitação a distância, utilizando interface tecnológica buscando maior adesão ao programa, maior eficácia na assimilação do conteúdo e cumprimento do exigido pela NR32, por exemplo. Cunha e Mauro (2010) descreveram o treinamento oferecido pelo Programa de Educação Continuada de uma instituição, segundo os trabalhadores de enfermagem, e analisaram como este treinamento poderia influenciar na implantação da NR32 em um hospital. Nesse estudo, dentre os achados evidenciados, destaca-se que os dados indicaram a necessidade de treinamento e formação específica na NR32 como importantes estratégias de implantação das medidas de proteção à saúde dos trabalhadores. Marziale et al. (2014) citam ainda, que em seu estudo, parte dos trabalhadores que sofreram acidente de trabalho em um hospital atribuiu a ocorrência do acidente a fatores individuais ou a colegas, mostrando este ser um indicador da necessidade da utilização de estratégias educativas e, principalmente, da conscientização desses trabalhadores quanto à necessidade de adoção de práticas seguras de trabalho e sobre os riscos ocasionados pelos acidentes, uma vez que muitos trabalhadores não identificaram as consequências da injúria sofrida quando acidentados com materiais biológicos. Em uma revisão integrativa, os autores afirmam que "dentre os trabalhos analisados, nota-se que uma ênfase para o papel da educação permanente juntos aos profissionais para a busca do êxito na implantação" mostram que estes aspectos são primordiais para obtenção de segurança e proteção dos profissionais da saúde (Santos Junior et al., 2015).

Santos et al. (2012) verificaram a implantação da NR32 em um hospital e percebeu-se que apesar das dificuldades encontradas na instituição (no âmbito de investimentos e negligência profissional, por exemplo), o mesmo atende certos quesitos em relação à NR32, em processo de implementação ou na sua totalidade, mostrando que adequar-se à norma não exige aporte financeiro das instituições, mas também a aderência dos trabalhadores, no que tange adotar atitude e comportamento seguros.
Atualmente, é possível observar que a qualidade de vida das pessoas no ambiente de trabalho é uma exigência geral. Nesse sentido, é preciso que elas estejam capacitadas para o enfrentamento dos acidentes de trabalho que surgem nos ambientes de trabalho, exigindo do profissional da área da saúde um olhar diferenciado para as questões sobre riscos e segurança no trabalho.

A NR32 define claramente as regras e condições de segurança e preservação da saúde dos trabalhadores que atuam nos ambientes de saúde. A referida norma trata de forma exclusiva sobre quais medidas que devem ser tomadas por empregadores e empregados para garantir a proteção dos profissionais dentro dos espaços de saúde, ressaltando o compartilhamento da responsabilidade de ambos sobre a execução da norma regulamentadora em questão. Entretanto, Morais et al. (2017), ao avaliar o conhecimento dos docentes enfermeiros de um curso de enfermagem sobre NR32, constatou que mais de $40 \%$ dos professores avaliados referiram não conhecer a norma, mostrando que mesmo com mais de uma década de publicação, a norma ainda não atinge todos os profissionais da saúde; e daqueles que conheciam a norma, os professores não mencionaram definições fundamentais desta norma. Outra análise que pode ser feita partir desse estudo é que alunos da área da saúde podem não estar sendo capacitados para atuarem com segurança dentro de seus laboratórios e posteriormente em seus locais de trabalho.

Com isso, este estudo possibilitou uma discussão mais profunda sobre a NR32, possibilitando levantar informações sobre aspectos relacionados com a saúde do trabaIhador, resguardando sua saúde e a própria assistência prestada aos usuários, tornando essa norma um instrumento que deve ser aplicado no cotidiano dos espaços de saúde.

Por acreditar nessa premissa, o referido trabalho propôs uma abordagem teórica a respeito da aplicação da NR32 nos espaços de trabalho dos trabalhadores da área da saúde e de que forma ocorre o gerenciamento dos riscos inerentes a saúde dos trabalhadores nos espaços de saúde. Pela ausência de estudos especificamente em laboratórios de instituições de ensino superior, faz-se necessário compreender os achados e transferi-los para a realidade encontrada por professores, técnicos e alunos.

Dentre vários benefícios encontrados ao longo da leitura dos artigos, a diminuição considerável das doenças ocupacionais foi citada em praticamente todos os estudos analisados, confirmando a importância da NR32 dentro dos ambientes de saúde. Quando relacionamos às instituições de ensino superior, fica claro que estes ambientes são responsáveis pela formação dos futuros profissionais 
da saúde e que estes deveriam sair de suas formações detendo o conhecimento sobre a legislação vigente a respeito de saúde e segurança no trabalho. Entretanto, ressalta-se novamente, que não foram localizadas pesquisas no qual laboratórios de saúde de instituições de ensino superior tenham sido o objeto de estudo.

\section{CONCLUSÃO}

Sendo assim, o estudo proposto não apresentou resultados quantitativos, pois a abordagem supracitada consistiu na discussão teórica no que tange a aspectos como aplicação da NR32, diminuição de riscos em ambientes de saúde e segurança do trabalho para os profissionais da saúde dentro da oferta dos serviços de saúde. Dentre os estudos analisados, apenas um deles tratava-se de uma revisão sobre a norma, porém com um número de artigos ínfimo (seis no total), mostrando a necessidade de aprofundamento de pesquisas que visem revisar os resultados obtidos através da implantação da NR32 (Santos Junior et al., 2015).

De forma mais específica, com a análise exploratória, obteve-se como resultados parciais algumas informações sobre a NR32 que reforçam a necessidade de inserção da referida norma em todos os ambientes de trabalho voltados para a manutenção da saúde, contribuindo para um ambiente laboral saudável para quem oferece esse tipo de serviço e melhorando os resultados das instituições. Ressalta-se ainda que por se tratar de um ambiente de educação, os laboratórios de saúde das instituições de ensino devem estar alinhados à norma para que os profissionais sejam formados com senso crítico apurado para questões referentes à SST, bem como oferecendo um ambiente seguro à comunidade acadêmica que os utilizem.

\section{REFERÊNCIAS}

Acácio, A. J. et al. 2013. Aplicabilidade da Norma Regulamentadora 32 (NR-32) e implicações para o enfermeiro do trabalho. Revista Eletrônica de Enfermagem do Centro de Estudos de Enfermagem e Nutrição 32, 32:1-16.

Agência Nacional de Vigilância Sanitária - Anvisa. 2003. Manual de Segurança no Ambiente Hospitalar. Brasília, DF: Anvisa.

Associação Brasileira de Enfermagem - Seção RJ, ABEn-RJ Cartilha do trabalhador de Enfermagem-Saúde, segurança e boas condições de trabalho, Rio de Janeiro, 2006. Disponível em: http://bvsms.saude.gov.br/bvs/publicacoes/cartilha_aben.pdf
Brasil. Ministério do Trabalho e Emprego. Portaria no 485, de 11 de novembro de 2005. Aprova a Norma Regulamentadora n.o 32 (Segurança e Saúde no Trabalho em Estabelecimentos de Saúde). Diário Oficial da União, Brasília, DF, 16 nov. 2005. Seção I.

Chizzotti, A. 2003. A pesquisa qualitativa em ciências humanas e sociais: evolução e desafios. Revista Portuguesa de Educação 16, 2:221-236. https://www.redalyc.org/ articulo.oa?id=37416210

Clock, D.; Batiz, E.C. 2017. Diagnóstico da implantação e implementação da Norma Regulamentadora 32 nos estabelecimentos de saúde: um estudo de caso em um hospital público de Joinville, SC - Brasil. Ação Ergonômica 11, 1:95-117. http://www.abergo.org.br/revista/index.php/ ae/article/view/699/282

Cremer, E. et al. 2013. Saúde do trabalhador e riscos de resíduo no ambiente hospitalar segundo a Norma Regulamentadora 32. Revista Salusvita 32, 3:265-284. http:// hdl.handle.net/11449/140720

Cunha, A.C.; Mauro, M.Y.C. 2010. Educação Continuada e a Norma Regulamentadora 32: utopia ou realidade na enfermagem? Revista Brasileira de Saúde Ocupacional 35, 122:305-313. https://doi.org/10.1590/ S0303-76572010000200013

Marconi M, Lakatos E. 2003. Fundamentos de metodologia científica. São Paulo: Atlas.

Marziale, M.H.P. et al. 2012. Implantação da Norma Regulamentadora 32 e o controle dos acidentes de trabalho. ACTA Paulista de Enfermagem 25, 6:859-866. https://doi. org/10.1590/S0103-21002012000600006

Marziale, M.H.P. et al. 2014. Consequences of occupational exposure to biological material among workers from a university hospital. Escola Anna Nery Revista de Enfermagem 18, 1:11-16. https://doi.org/10.5935/14148145.20140002

Marziale, M.H; Carvalho, E.C. 1998. Condições ergonômicas do trabalho da equipe de enfermagem em unidade de internação de cardiologia. Revista Latino-Americana de Enfermagem 6, 1:99-117. https://doi.org/10.1590/ S0104-11691998000100013

Morais, R.L.G.L. et al. 2017. Conhecimentos e condutas de biossegurança entre docentes de enfermagem. Revista Online de Pesquisa Cuidado é Fundamental 9, 1:137-143. http://dx.doi.org/10.9789/2175-5361.2017.v9i1.137-143 
Oliveira, J.S. et al. 2015. Acidentes com perfurocortantes entre trabalhadores de saúde. Revista de APS 18, 1:108-115.

Pustiglione, M. et al. 2015. Modelo e-learning de capacitação na Norma Regulamentadora no 32 (NR 32). Revista Brasileira de Medicina do Trabalho 12, 1:39-42.

Robazzi, M.L.C.C; Marziale, M.H.P. 2004. A norma regulamentadora 32 e suas implicações sobre os trabaIhadores de enfermagem. Revista Latino-Americana de Enfermagem 12, 5:834-836. http://dx.doi.org/10.1590/ S0104-11692004000500019

Santos Junior, A.G. et al. 2015. Norma Regulamentadora 32 no Brasil: revisão integrativa de literatura. Revista de Enfermagem do Centro Oeste Mineiro 5, 1:1528-1534. https://doi.org/10.19175/recom.v0i0.1050

Santos, M.R. et al. 2012. Avaliação da implantação da Norma Regulamentadora 32 em um hospital universitário. Revista Cogitare Enfermagem 17, 3:524-530. http:// dx.doi.org/10.5380/ce.v17i3.25754

Silva, E.F. 2018. A importância da Norma Regulamentadora 32 nos serviços de prestação de saúde. São Paulo: LTr.

Silva, V.G. et al. 2015. Relato de experiência do treinamento em serviço sobre a Norma Regulamentadora - NR 32. Revista Rede de Cuidados em Saúde 9, 2.

Recebido: 28 set. 2020

Aprovado: 02 nov. 2020

DOI: 10.20985/1980-5160.2020.v15n3.1678

Como citar: Fracasso, B.V.; Libânio, C.S.; Amaral, F.G. (2020). NR32: revisão à proteção em laboratórios de instituições de ensino superior. Revista S\&G 15, 3, 294-300. https://revistasg.emnuvens.com.br/sg/article/ view/1678 